\title{
The relationship between muscular atrophy/sarcopenia and cardiovascular diseases in the elderly: a bibliometrics study
}

\author{
Zhisheng Tan ${ }^{1 \#}$, Yunchun Zhao ${ }^{1 \#, ~ Z h e n g m i n ~ J i n ~}{ }^{1}$, Genyu $\mathrm{Li}^{1}, \mathrm{Li} \mathrm{Xu}^{1}$, Wenli $\mathrm{Li}^{1}$, Yunhua Liang ${ }^{1}$, \\ Shuying Wang ${ }^{2}$, Qin Zhu ${ }^{1}$ \\ ${ }^{1}$ Cadre Ward, 920th Hospital of Joint Logistics Support Force of PLA, Kunming, China; ${ }^{2}$ Department of Blood Transfusion, First Medical Center of \\ PLA General Hospital, Beijing, China \\ Contributions: (I) Conception and design: Z Tan, Q Zhu; (II) Administrative support: Y Zhao, Z Jin; (III) Provision of study materials or patients: G \\ Li, L Xu; (IV) Collection and assembly of data: Z Tan, W Li; Y Liang; (V) Data analysis and interpretation: Z Tan, S Wang, Q Zhu; (VI) Manuscript \\ writing: All authors; (VII) Final approval of manuscript: All authors. \\ \#These authors contributed equally to this work. \\ Correspondence to: Qin Zhu. Cadre Ward, 920th Hospital of Joint Logistics Support Force of PLA, 212 Daguan Road, Xishan District, Kunming \\ 650032, China. Email: zq11302021@163.com.
}

Background As the aging population continues to increase worldwide, the prevalence of cardiovascular diseases and muscular dystrophy/sarcopenia in the elderly has escalated significantly. Cardiovascular diseases elevate the risk of muscular atrophy/sarcopenia, which results in increased disability and mortality of patients. This study analyzed the current available literature related to the relationship between cardiovascular diseases and muscular atrophy/sarcopenia in the aging population.

Methods: The Science Citation Index Expanded (SCI-E) database was searched for related literature published between 1900 and March 14, 2021. The subject search was performed using the search terms "muscular atrophy" and "sarcopenia". The search formula was "muscular atrophy OR sarcopenia". The search scope was limited to "cardiovascular diseases OR cardiac \& cardiovascular systems". All search results and cited references were exported in plain text format and Citespace software was used to analyze the publications in terms of year of publication, country and institution, journal of publication, authors, and keywords.

Results: A total of 1,004 related research documents were obtained, with a citation frequency of 26,705 times. The top five countries for the highest number of published documents were the United States, Japan, Germany, South Korea, and Italy. The top five countries involved in research cooperation were the United States, Japan, the United Kingdom, Spain, and Germany, however, overall, there was little cooperation between countries, institutions, and authors. A number of researchers from Germany published the most documents. The author with the most cited publication was Cruz-Jentoft et al. from Spain, which deserves special attention. Professional journals of in the field of geriatrics play a significant role in this research topic. Analysis of the keywords showed that current researchers are mainly concerned with the associated risk of death.

Conclusions: The relationship between muscular atrophy/sarcopenia and cardiovascular diseases is currently a hot topic of research in geriatrics and cardiovascular disease, and further studies examining the mechanisms involved and potential prevention strategies are warranted.

Keywords: Muscular atrophy; sarcopenia; cardiovascular diseases; elderly; bibliometrics study

Submitted Jun 07, 2021. Accepted for publication Aug 12, 2021.

doi: 10.21037/apm-21-2144

View this article at: https://dx.doi.org/10.21037/apm-21-2144 


\section{Introduction}

Improvements in the standard of living and advancements in medicine have increasingly led to an aging society in many countries. According to China's seventh national census published on May 11, 2021, there are over 264.02 million people aged 60 years and over in China, accounting for $18.70 \%$ of whole population. Furthermore, the population of people aged 65 years and over is 190.64 million, accounting for $13.50 \%$ of the population. Compared with 2010, the proportion of the population aged 60 and over has increased by $5.44 \%$ (1). In the 27 European Union (EU) member states, the proportion of the population aged 65 years and over was about $20.3 \%$ in 2019 and is expected to reach $24.2 \%$ by 2030 . The 2019 and projected 2030 figures are $18.5 \%$ and $21.5 \%$, respectively, for the United Kingdom; $16.2 \%$ and $20.3 \%$, respectively for the United States; $28.0 \%$ and $30.95 \%$, respectively for Japan; and $15.1 \%$ and $24.7 \%$, respectively for South Korea (2). Therefore, aging in the major countries is accelerating rapidly. In fact, many medical studies in recent years have changed the classification standard for the elderly from the previous 60 years to the current 65 years (3). Age is an independent risk factor for many diseases, especially cardiovascular disease. An increase in age elevates the risk of hypertension, coronary heart disease, myocardial infarction, heart failure, and death (4-7). Concurrently, the intake of nutrients by the elderly tends to decrease, often resulting in muscle atrophy (8-12). Sarcopenia is a clinical syndrome characterized by progressive and extensive decline in skeletal muscle mass and hypofunction (13). In October 2016, the U.S. Centers for Disease Control and Prevention established the ICD-10-CM code (M62.84) for sarcopenia. Sarcopenia is defined as a dysfunction caused by decreased skeletal muscle mass and strength in the elderly $(8,14)$. Interestingly, cardiovascular disease is closely related to muscle wasting and muscle reduction (15), and both cardiovascular disease and muscle atrophy/ muscle reduction can seriously affect the quality of life of elderly patients and increase the risk of morbidity and mortality $(16,17)$. Especially in patients with heart failure, the incidence of sarcopenia is high and these two conditions often deteriorate each other (18). For now, most scholars recommend enough nutrition and reasonable physical exercise are benefit for prevention of muscular atrophy/ sarcopenia (19). Bibliometrics provides a method to analyze the current literature in a certain field, thereby imparting an important reference point for researchers (20). This current study examined the general situation of research in the field of muscular atrophy/sarcopenia and cardiovascular diseases.

\section{Methods}

\section{Data source}

The Science Citation Index Expanded (SCI-E) database is the core data set in the Web of Science. It includes the titles of papers published by important journals in the field of natural sciences and their cited information. SCI-E is an important tool for bibliometrics and scientific research evaluation. This research used SCI-E as the data source to search relevant literature. The target publication time of the literature was not limited, and thus, included literature published from 1900 to the time of this study, that is, March 14, 2021.

\section{Strategy of the literature search}

In this study, the subject search was used. The search terms were "muscular atrophy" and "sarcopenia", and the search formula was "muscular atrophy OR sarcopenia". The search scope was limited to "cardiovascular diseases OR cardiac \& cardiovascular systems".

\section{Analysis}

Records of all the search results and cited references were exported in plain text format and Citespace software was used to analysis the year of publication, the country and institution of publication, the journal of publication, the authors, and the use of keywords. The results reflect the current research status of the relationship between muscle reduction, muscle atrophy, and cardiovascular disease in the field of geriatrics.

\section{Statistical analysis}

This research is a descriptive study. The ratio (percentage) of each indicator reflects the distribution and changing trends of different years, countries, institutions, journals, and authors without statistical comparison. Conference abstracts, conference documents, and duplicate revision documents were excluded.

\section{Results}

\section{General information}

A total of 1,040 articles were obtained from the database 
Table 1 An analysis of the document types in the literature

\begin{tabular}{lcc}
\hline Literature type & Records & Percentage (\%) \\
\hline Article & 668 & 66.5 \\
Review & 215 & 21.4 \\
Meeting Abstract & 70 & 7.0 \\
Editorial Material & 29 & 2.9 \\
Letter & 21 & 2.1 \\
Proceedings Paper & 20 & 2.0 \\
Book Chapter & 8 & 0.8 \\
Early Access & 8 & 0.8 \\
Correction & 1 & 0.1 \\
\hline
\end{tabular}

search, including 668 original articles, 215 review articles, 70 meeting abstracts, 29 editorial materials, 21 letters, 20 proceeding articles, 8 books, 8 articles of early access and 1 correction. There was 1 correction publication and 36 duplicate literature. After exclusions, a total of 1,004 articles were included in this investigation (Table 1). Analysis of the number of documents published each year revealed an accelerated growth trend, especially after 2009 (Table 2, Figure 1). These papers were cited 28,142 times in total, with an h-index of 83 , and the average number of citations per literature was 28.0 (Figure 2).

\section{Distribution of countries and institutions}

The results showed that the top 5 countries with the greatest number of publications were the United States, Japan, Germany, South Korea, and Italy. The top 5 countries in terms of research cooperation were the United States, Japan, the United Kingdom, Spain, and Germany (Tables 3,4). The following institutions published more than 20 articles: the Charité-Universitätsmedizin Berlin, University Medical Center Göttingen, and Yonsei University. The 4 institutions with the highest centrality scores (which implies increased cooperation) were CharitéUniversitätsmedizin Berlin, Duke University, National Institute on Aging (NIA) and Columbia University (Tables 5,6). The CiteSpace $\mathrm{V}$ software was used to generate the visualization atlas of the countries (Figure 3) and institutions of publication (Figure 4). The main countries researching this field were all developed countries with an obvious aging population, especially the United States and Japan. Although there was some cooperation between institutions, it can be
Table 2 The distribution of literature according to publication year

\begin{tabular}{|c|c|c|}
\hline Publication year & Records & Percentage (\%) \\
\hline 2021 & 23 & 2.3 \\
\hline 2020 & 205 & 20.4 \\
\hline 2019 & 163 & 16.2 \\
\hline 2018 & 127 & 12.6 \\
\hline 2017 & 97 & 9.7 \\
\hline 2016 & 77 & 7.7 \\
\hline 2015 & 60 & 6.0 \\
\hline 2014 & 45 & 4.5 \\
\hline 2013 & 41 & 4.1 \\
\hline 2012 & 28 & 2.8 \\
\hline 2011 & 21 & 2.1 \\
\hline 2010 & 20 & 2.0 \\
\hline 2009 & 10 & 1.0 \\
\hline 2008 & 20 & 1.0 \\
\hline 2007 & 15 & 1.5 \\
\hline 2006 & 9 & 0.9 \\
\hline 2005 & 4 & 0.4 \\
\hline 2004 & 6 & 0.6 \\
\hline 2003 & 4 & 0.4 \\
\hline 2002 & 2 & 0.2 \\
\hline 2001 & 4 & 0.4 \\
\hline 2000 & 3 & 0.3 \\
\hline 1999 & 4 & 0.4 \\
\hline 1998 & 4 & 0.4 \\
\hline 1997 & 3 & 0.3 \\
\hline 1996 & 1 & 0.1 \\
\hline 1995 & 3 & 0.3 \\
\hline 1994 & 1 & 0.1 \\
\hline 1993 & 1 & 0.1 \\
\hline 1991 & 3 & 0.3 \\
\hline
\end{tabular}

seen that the nodes are relatively scattered.

\section{Author analysis}

At the time of this study, the authors with more than 10 publications included Von Haehling S, Anker SD, 


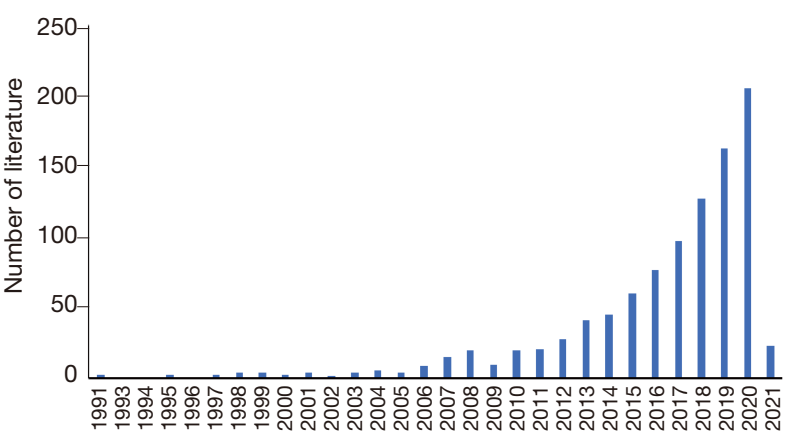

Figure 1 Number of publications per year.

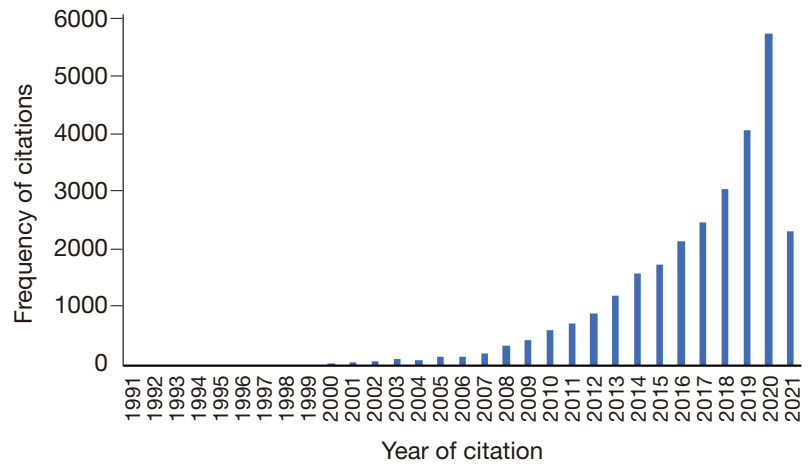

Figure 2 Number of citations per year.

Table 3 The top 10 countries in terms of the number of publications in this field

\begin{tabular}{lcc}
\hline Rank & Countries & Frequency \\
\hline 1 & USA & 243 \\
2 & Japan & 172 \\
3 & Germany & 106 \\
4 & South Korea & 91 \\
5 & Italy & 85 \\
6 & China & 62 \\
7 & Canada & 59 \\
8 & England & 50 \\
9 & Brazil & 49 \\
10 & Spain & 38 \\
\hline
\end{tabular}

Table 4 The top 10 countries for centrality

\begin{tabular}{lcc}
\hline Rank & Countries & Centrality \\
\hline 1 & USA & 0.53 \\
2 & Japan & 0.25 \\
3 & England & 0.19 \\
4 & Spain & 0.17 \\
5 & Germany & 0.15 \\
6 & Italy & 0.12 \\
7 & Brazil & 0.12 \\
8 & Canada & 0.10 \\
9 & Australia & 0.08 \\
10 & China & 0.06 \\
\hline
\end{tabular}

Table 5 The top 10 institutions in terms of the number of publications

\begin{tabular}{lcc}
\hline Rank & Institutions & Frequency \\
\hline 1 & Charite Univ Med Berlin & 33 \\
2 & Univ Med Ctr Gottingen & 21 \\
3 & Yonsei Univ & 21 \\
4 & Univ Sao Paulo & 15 \\
5 & Sungkyunkwan Univ & 12 \\
6 & National Institute on Aging & 11 \\
7 & Kitasato Univ Hosp & 10 \\
8 & Johns Hopkins Univ & 10 \\
9 & Univ Melbourne & 9 \\
10 & DZHK German Ctr Cardiovasc Res & 9 \\
\hline
\end{tabular}

Table 6 The top 10 institutions by centrality

\begin{tabular}{lcc}
\hline Rank & Institutions & Centrality \\
\hline 1 & Charite Univ Med Berlin & 0.08 \\
2 & Duke Univ & 0.08 \\
3 & National Institute on Aging & 0.07 \\
4 & Columbia Univ & 0.07 \\
5 & Korea Univ & 0.06 \\
6 & Univ Pittsburgh & 0.06 \\
7 & Kyung Hee Univ & 0.06 \\
8 & McGill Univ & 0.05 \\
9 & Sungkyunkwan Univ & 0.04 \\
10 & Inje Univ & 0.04 \\
\hline
\end{tabular}




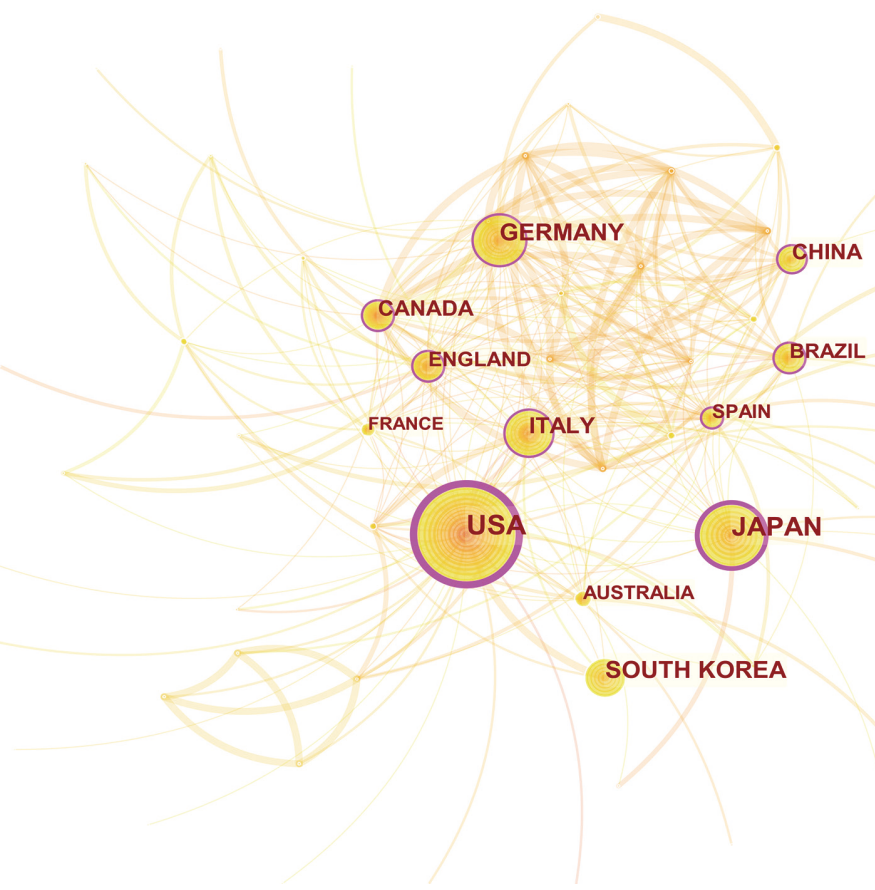

Figure 3 A visual map of cooperation between countries.

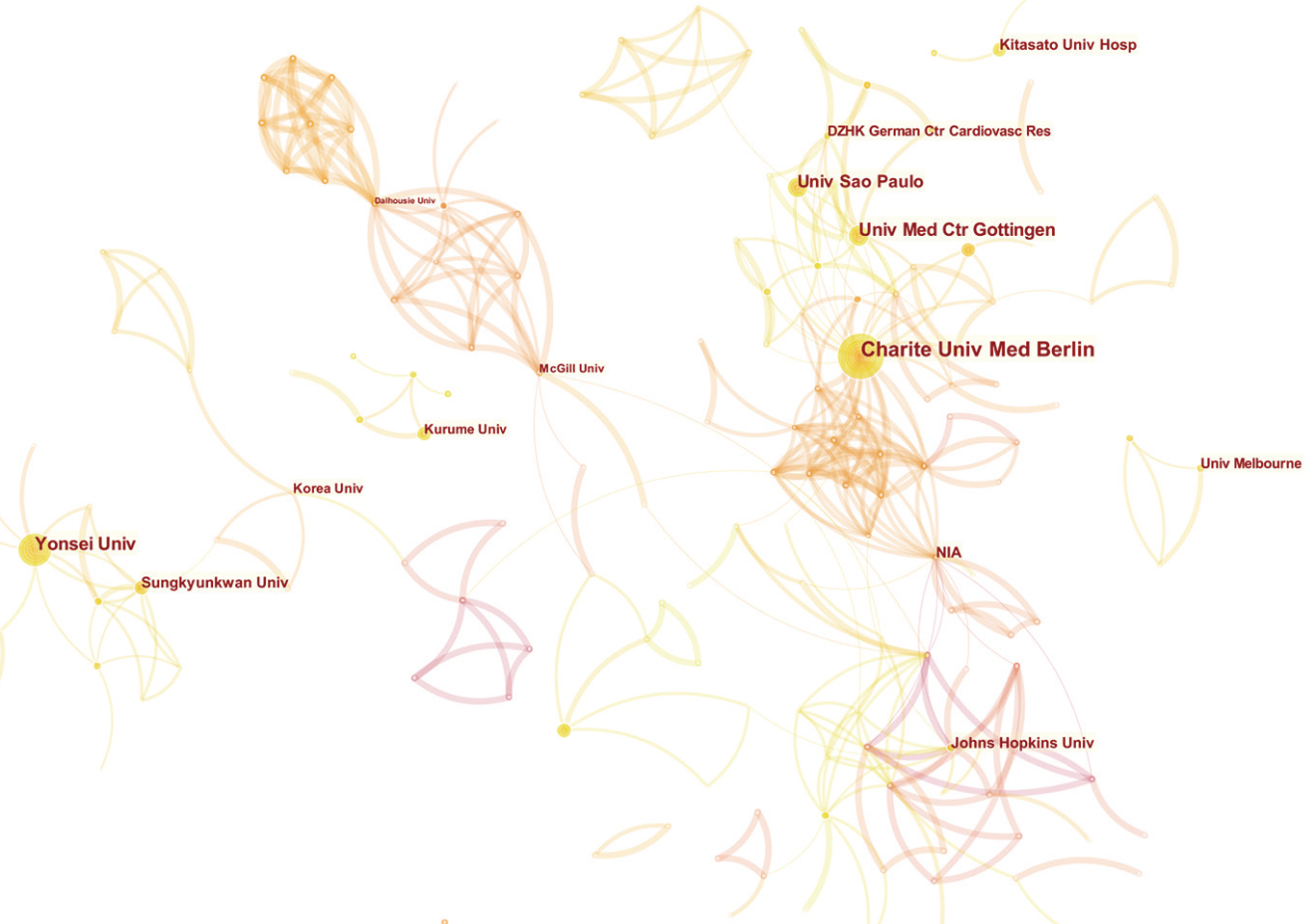

Figure 4 A visual map of the cooperation relationship between institutions. 
Table 7 The top 10 authors by number of publications

\begin{tabular}{lcc}
\hline Rank & Authors & Frequency \\
\hline 1 & Von Haehling S & 30 \\
2 & Anker SD & 25 \\
3 & Springer J & 15 \\
4 & Doehner W & 13 \\
5 & Matsunaga A & 9 \\
6 & Nozaki K & 8 \\
7 & Ako J & 8 \\
8 & Hamazaki N & 8 \\
9 & Maekawa E & 8 \\
10 & Matsuzawa R & 8 \\
\hline
\end{tabular}

Springer J, and Doehner W. In fact, these four authors are all from Germany, and with the exception of Doehner W who is from Charité-Campus Virchow, all others are from the University Medical Centre Göttingen. A similar distribution of institutions was observed. In this study, no author centrality score reached 0.01 , indicating that there was relatively little collaboration between authors from different institutions (Table 7, Figure 5). There were 6 authors who have been cited more than 100 times, including Cruz-Jentoft AJ, Baumgartner RN, Janssen I, Morley JE, Fried LP, and Chen LK (Table 8). Further analysis showed that in this field, Professor Cruz-Jentoft AJ often published important papers in top journals with an extremely high frequency of citations. Professor Baumgartner RN's basic research mainly focused on the body composition of the human being within the field of muscular atrophy and sarcopenia, and is also often cited. The top 3 authors by centrality were Baumgartner RN, Anker SD, and Morley JE (Table 9, Figure 6).

\section{Fournal distribution}

The 1,004 documents retrieved from this database search covered 426 journals, all of which are widely distributed. Among them, there were 17 journals with more than 10 articles (Table 10). These journals published 264 articles in this field, accounting for $26.3 \%$ of the total number
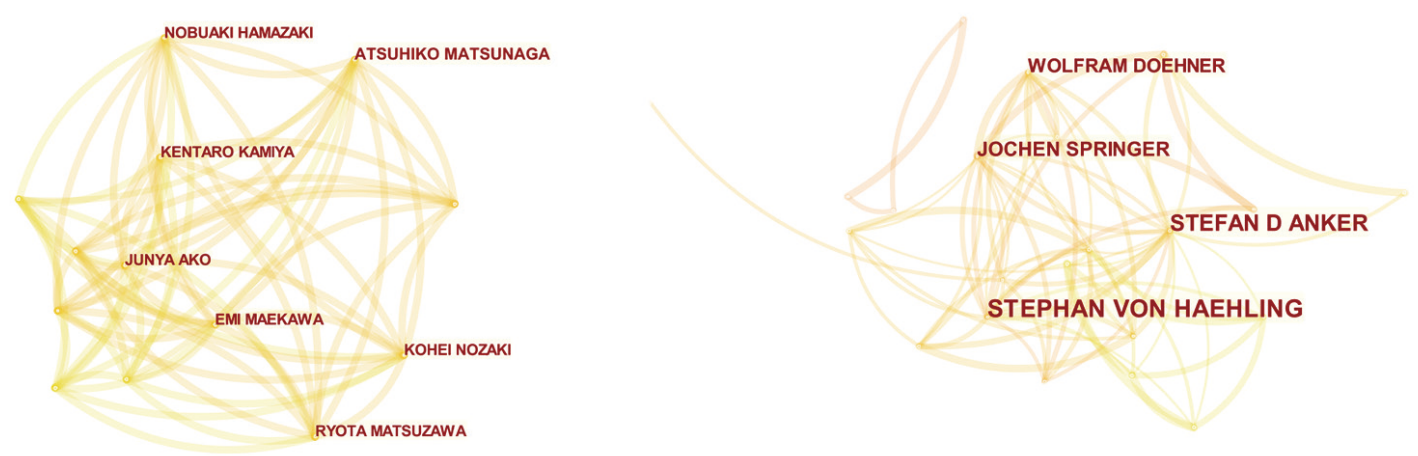

Figure 5 A visual map of the collaboration between authors. The grid at bottom represents authors with fewer collaboration compared with authors above (the software didn't show the authors' name automatically). 
Table 8 The top 10 most cited authors by frequency of citations

\begin{tabular}{lcc}
\hline Rank & Authors & Frequency \\
\hline 1 & Cruz-Jentoft AJ & 303 \\
2 & Baumgartner RN & 153 \\
3 & Janssen I & 149 \\
4 & Morley JE & 146 \\
5 & Fried LP & 116 \\
6 & Chen LK & 106 \\
7 & Newman AB & 99 \\
8 & Visser M & 93 \\
9 & Von Haehling S & 93 \\
10 & Cesari M & 92 \\
\hline
\end{tabular}

Table 9 The top 10 cited authors by centrality

\begin{tabular}{lcc}
\hline Rank & Authors & Centrality \\
\hline 1 & Baumgartner RN & 0.11 \\
2 & Anker SD & 0.10 \\
3 & Morley JE & 0.09 \\
4 & Busquets S & 0.08 \\
5 & Visser M & 0.08 \\
6 & Bhasin S & 0.07 \\
7 & Aubertin-Leheudre M & 0.05 \\
8 & Blair SN & 0.05 \\
9 & Coats AJS & 0.05 \\
10 & Adams V & 0.04 \\
\hline
\end{tabular}

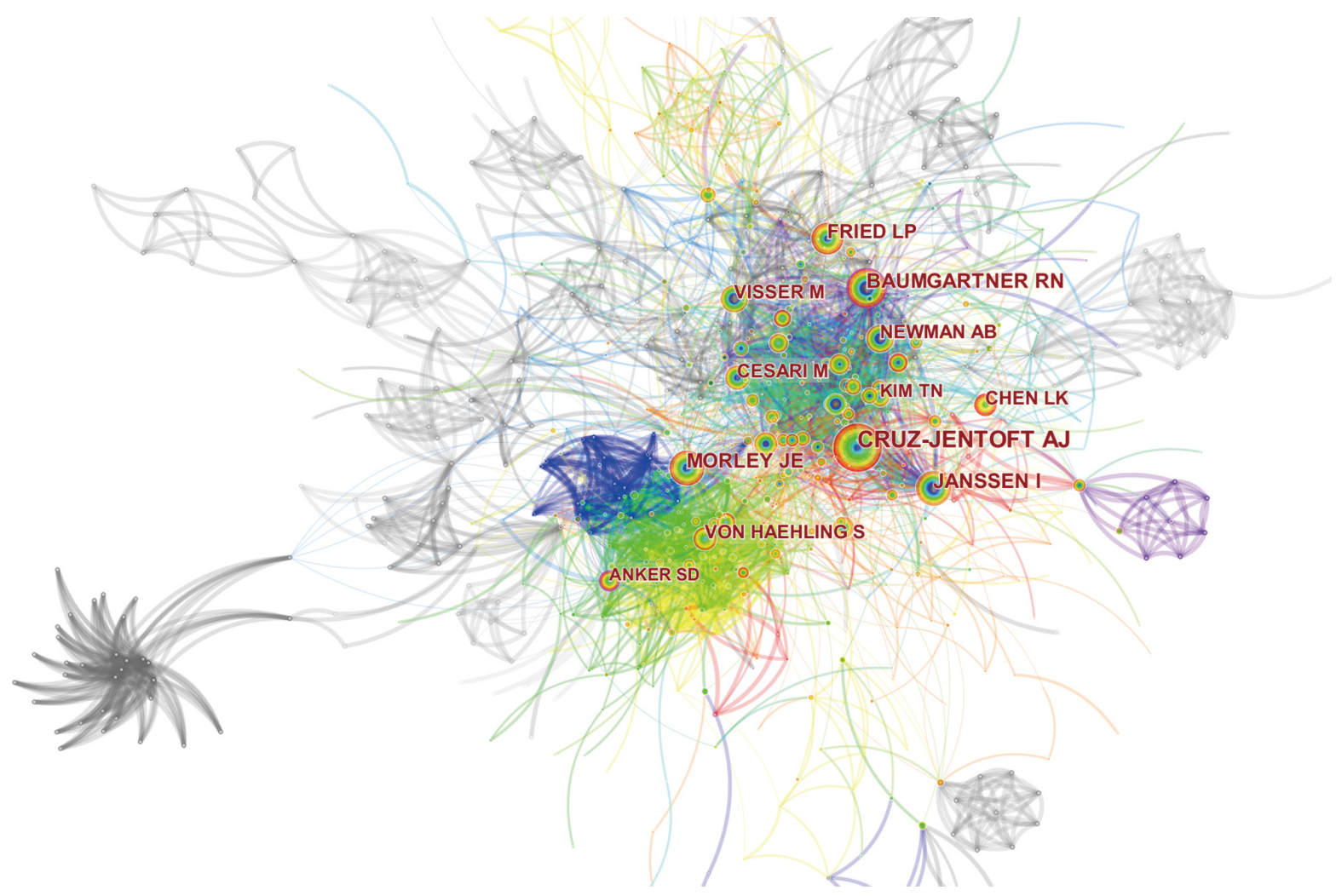

Figure 6 A visual map of co-cited authors.

of documents (Table 10). The top 6 journals with the greatest number of published papers in this field were all cardiovascular journals (Table 10). However, the top 5 journals with the highest citation frequency only included one cardiovascular journal, and the others were geriatric journals and related professional journals (Table 11). The top 3 journals with the highest centrality scores were American Fournal of Medicine, American Fournal of Pathology, and Circulation (Table 12). A comparison of the number of citations (Table 11) and the number of articles (Table 10), revealed that the top 10 journals according to the number of citations, with the exception of Circulation, had fewer 
Table 10 The top 17 journals by number of publications in this field

\begin{tabular}{|c|c|c|c|}
\hline Rank & Journals & Records & Percentage $(\%)$ \\
\hline 2 & Circulation & 25 & 2.5 \\
\hline 3 & Esc Heart Failure & 24 & 2.4 \\
\hline 4 & European Heart Journal & 18 & 1.8 \\
\hline 6 & Journal of The American College of Cardiology & 17 & 1.7 \\
\hline 7 & Journal of Nutrition Health Aging & 15 & 1.5 \\
\hline 8 & Journal of Heart and Lung Transplantation & 14 & 1.4 \\
\hline 9 & PLoS One & 14 & 1.4 \\
\hline 12 & J Gerontol A-Biol & 12 & 1.2 \\
\hline 13 & Journal of Cardiology & 11 & 1.1 \\
\hline 14 & Nutrition Metabolism and Cardiovascular Diseases & 11 & 1.1 \\
\hline 15 & Scientific Reports & 11 & 1.1 \\
\hline 16 & Experimental Gerontology & 10 & 1.0 \\
\hline 17 & Journal of The American Medical Directors Association & 10 & 1.0 \\
\hline
\end{tabular}

Table 11 The top 10 journals by citation frequency

\begin{tabular}{lcc}
\hline Rank & Journals & Frequency \\
\hline 1 & Journals of Gerontology Series A: Biological Sciences And Medical Sciences & 437 \\
2 & Age Ageing & 396 \\
3 & Circulation & 391 \\
4 & Journal of American Geriatric Society & 388 \\
5 & Am J Clinical Nutrition & 357 \\
6 & PLoS One & 347 \\
7 & New England Journal of Medicine & 333 \\
9 & Lancet & 333 \\
10 & Journal of The American College of Cardiology & 301 \\
\hline
\end{tabular}

published articles. This suggested that the average number of citations of these journals was high (for example, the 12 articles in J Gerontol A-Biol had a citation frequency of 437 times, with an average of 36.4 times per article), and thus, its academic value may be higher.
Keywords reflect the research botspots and frontiers in this field

CiteSpace V software was used to generate a keyword cooccurrence map (Figure 7). The keyword statistical analysis results showed that the top 5 commonly used keywords 
Table 12 The top 10 journals by centrality

\begin{tabular}{lcc}
\hline Rank & Journals & Centrality \\
\hline 1 & American Journal of Medicine & 0.11 \\
2 & American Journal of Pathology & 0.08 \\
3 & Circulation & 0.07 \\
4 & American Journal of Cardiology & 0.06 \\
5 & Anerican Journal of Physiology-Endocrinology and Metabolism & 0.05 \\
6 & Archive of Internal Medicine & 0.05 \\
8 & Journal of Biological Chemistry & 0.05 \\
10 & American Heart Journal & 0.05 \\
\hline
\end{tabular}

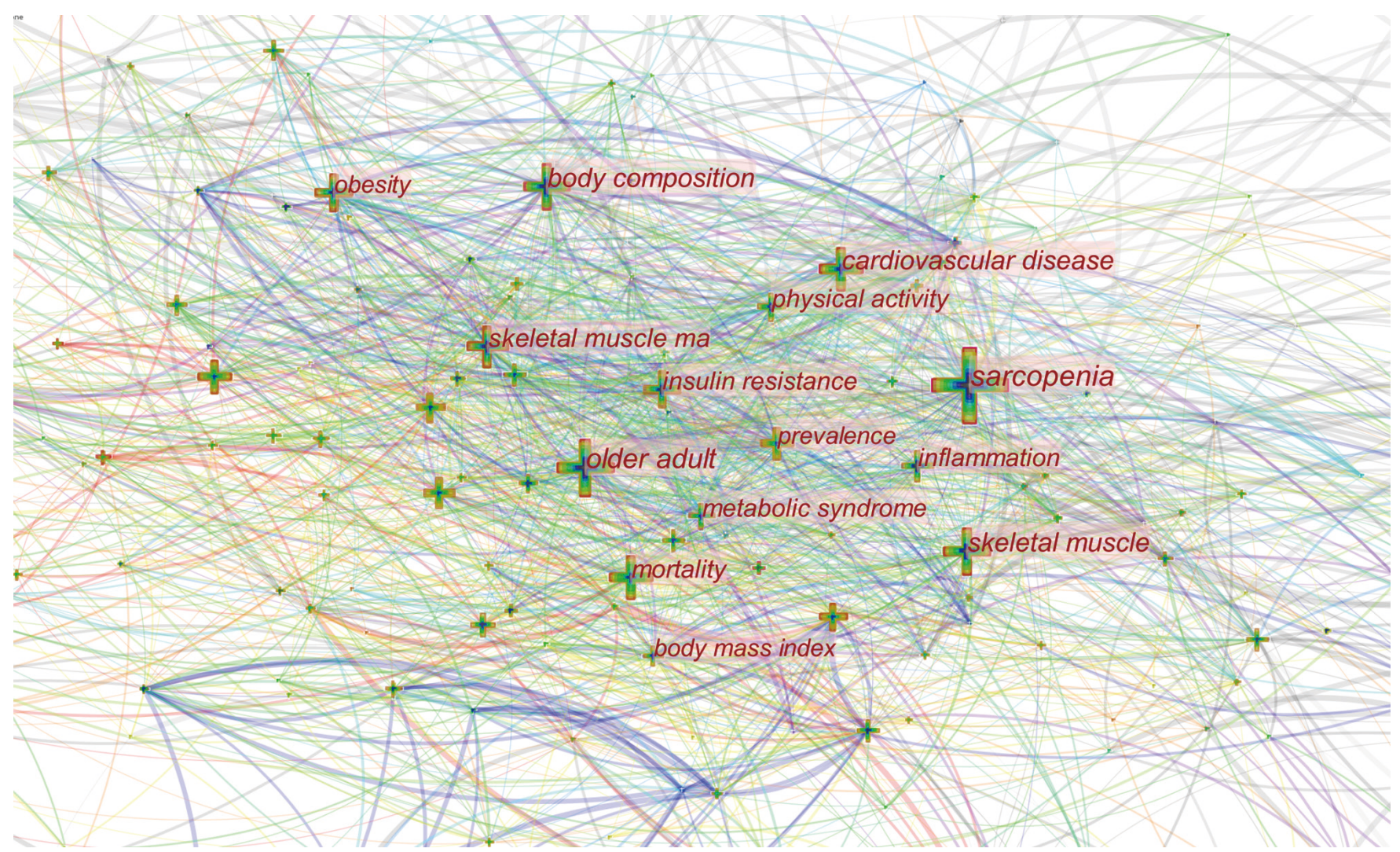

Figure 7 Keyword co-occurrence map. 
Table 13 The top 10 most frequently used keywords

\begin{tabular}{lcc}
\hline Rank & Keywords & Frequency \\
\hline 1 & Sarcopenia & 550 \\
2 & Older adult & 262 \\
3 & Cardiovascular disease & 202 \\
4 & Skeletal muscle & 196 \\
5 & Mortality & 186 \\
6 & Body composition & 165 \\
7 & Obesity & 131 \\
8 & Skeletal muscle ma & 130 \\
9 & Frailty & 118 \\
10 & Risk & 107 \\
\hline
\end{tabular}

Table 14 The top 10 keywords for centrality

\begin{tabular}{lrc}
\hline Rank & Keywords & Centrality \\
\hline 1 & Sarcopenia & 0.19 \\
2 & Older adult & 0.15 \\
3 & Skeletal muscle & 0.14 \\
4 & Body composition & 0.13 \\
5 & Cardiovascular disease & 0.11 \\
6 & Disability & 0.10 \\
7 & Chronic heart failure & 0.07 \\
8 & Spinal muscular atrophy & 0.07 \\
9 & Acute myocardial infarction & 0.07 \\
10 & Skeletal muscle ma & 0.06 \\
\hline
\end{tabular}

were sarcopenia, older adult, cardiovascular disease, skeletal muscle, and mortality. The centrality ranking results were similar (see Tables 13 and 14 for details). CiteSpace was then used to perform burst detection on high-frequency keywords, and the results are shown in Figure 8.

\section{Discussion}

The SCI-E database was searched to obtain relevant literature on muscular atrophy/sarcopenia and cardiovascular diseases. This study revealed that the number of publications in this field has been escalating in recent years, showing that the medical community is increasingly concerned about related issues. Analysis of the sources of the literature revealed that the countries that conducted the most research in this field were mainly developed countries in Europe, the United States, and Japan and South Korea in Asia. The main research institutions were also from these countries, but the cooperation between institutions and countries was relatively limited. The analysis of collaboration was based on few guidelines and consensus $(8,21,22)$. A number of researchers from Germany have published a significant number of documents. CruzJentoft AJ from Spain was the most cited author and is an important researcher in this field. Many articles have been published in top journals and are worthy of attention (23), in particular, journals in the field of geriatrics. The keyword analysis results showed that a main concern of current researchers is the associated risk of death.

Considering the population growth trend of numerous countries in recent years, the accelerated increase in the number of publications after 2009 is likely to be closely related to the aging society of countries worldwide, especially Japan, South Korea, and several developed countries in Europe and America. As the life expectancy of the elderly is gradually prolonged, the prevalence of various chronic diseases is also expected to gradually increase, especially cardiovascular disease, which is currently the leading cause of death in developed countries (24). Despite improvements in living conditions and medical advances, there are still many elderly people who suffer from muscle atrophy and muscle loss due to various reasons $(25,26)$. These factors have led to a large number of elderly people presenting with both muscular atrophy/sarcopenia and cardiovascular diseases. Insufficient blood supply to the heart or decreased heart function, coupled with atrophy/ decrease of muscle tissue, leads to a significant decline in the quality of life of these elderly people, and at the same time increases the risk of falling $(13,27,28)$. In the past, we often use "frailty", "muscular atrophy" to describe the status of old people with sarcopenia. Until 2016, ICD-10-CM give sarcopenia a code of M62.84 and a clear definition (29). Compared with old people, young patients with sarcopenia are often on dialysis or have comorbid disease, such as cancer, tuberculosis and inflammatory bowel disease (30-32). Sarcopenia can also inflict significant burden in terms of medical expenses (33). Therefore, relevant research into the prevention and treatment of muscular atrophy/sarcopenia complicated with cardiovascular diseases is warranted, so as to improve the quality of life of elderly patients and reduce the risk of disability and mortality. Besides cardiovascular diseases, other diseases, including chronic obstructive 


Keywords
disability
women
older men
inflammation
cardiovascular disease risk
older person
metabolic syndrome
postmenopausal women
adipose tissue
cancer cachexia
alzheimers disease
insulin resistance
population
sarcopenic obesity
chronic heart failure
national health
epidemiology
malnutrition
computed tomography

\begin{tabular}{rrrr} 
Year & Strength Begin End & 1991-2021 \\
1991 & 5.23 & 1995 & 2009 \\
1991 & 3.69 & 2001 & 2008 \\
1991 & 4.6 & 2007 & 2012 \\
1991 & 4.33 & 2007 & 2011 \\
1991 & 6.68 & 2010 & 2016 \\
1991 & 5.68 & 2010 & 2013 \\
1991 & 7.88 & 2011 & 2016 \\
1991 & 3.61 & 2012 & 2016 \\
1991 & 4.04 & 2013 & 2015 \\
1991 & 3.78 & 2013 & 2016 \\
1991 & 3.68 & 2013 & 2015 \\
1991 & 6.47 & 2014 & 2016 \\
1991 & 3.71 & 2015 & 2017 \\
1991 & 3.55 & 2015 & 2016 \\
1991 & 3.47 & 2015 & 2017 \\
1991 & 4.13 & 2016 & 2018 \\
1991 & 3.98 & 2017 & 2019 \\
1991 & 3.99 & 2018 & 2019 \\
1991 & 3.58 & 2018 & 2021 \\
\hline
\end{tabular}

Figure 8 The top 19 keywords with the strongest citation bursts.

pulmonary diseases, cancers and renal diseases are all often seen with sarcopenia in the elderly (23).

Our analysis revealed that there is little cooperation between researchers in this field, and this may be related to the different living habits and causes of aging in various countries and regions. Indeed, the diet and living habits of the European Mediterranean countries, the United States, Japan, and South Korea are vastly different (34). Second, in an aging country, the elderly forms a large proportion of the population and thus, it is relatively easy to meet the sample size requirements of research without having to collaborate with other research groups. In addition, the welfare benefits and medical insurance systems for the elderly in different countries vary greatly (35). Especially in developing or poor countries, muscular atrophy/sarcopenia is very common in the elderly (26). Unfortunately, few studies in this field have examined the mechanisms involved and potential treatments. Investigating the relationship between sarcopenia and cardiovascular diseases in elderly patients is vital and should involve inter-institutional and inter-country cooperation.

To date, research into the relationship between muscular atrophy/sarcopenia and cardiovascular diseases has revealed several key findings. First, patients with sarcopenia obesity have an increased incidence of cardiovascular disease and mortality, especially heart failure (36). Experts have come to a consensus that sarcopenia is significantly prevalent in patients with heart failure, resulting in its poor prognosis (37). Second, skeletal muscle reduction is an important factor in the occurrence of sarcopenia in patients with cardiovascular disease (38). Third, in patients with sarcopenia, the main mechanisms of heart failure include the ubiquitin-protease system, autophagy, apoptosis, inflammation, and oxidative stress (37). For patients with muscular atrophy/sarcopenia, the main form of treatment is reasonable nutritional support. For patients with cardiovascular diseases, exercise rehabilitation is the treatment of choice for the prevention and treatment of sarcopenia. Unfortunately, to date, there has been little medical breakthrough in terms of treatment regimens $(37,39)$.

Therefore, it is recommended that future related studies further explore the mechanisms, sports rehabilitation, drug treatment options, and other early preventive measures in elderly patients with atrophy/sarcopenia and cardiovascular disease.

There were some limitations to this study. First, the concept of muscular atrophy/sarcopenia has undergone some changes and this may have caused the omission of some early literature. However, the inclusion of the 
publication by Baumgartner et al. in our search results suggested that important early documents were not omitted. Second, some basic research articles may not have been classified as cardiovascular disease-related literature and may have been omitted. Future research should include in-depth analysis of the citations listed in the related documents to supplement the search results.

\section{Acknowledgments}

Funding: None.

\section{Footnote}

Conflicts of Interest: All authors have completed the ICMJE uniform disclosure form (available at https://dx.doi. org/10.21037/apm-21-2144). The authors have no conflicts of interest to declare.

Ethical Statement: The authors are accountable for all aspects of the work in ensuring that questions related to the accuracy or integrity of any part of the work are appropriately investigated and resolved.

Open Access Statement: This is an Open Access article distributed in accordance with the Creative Commons Attribution-NonCommercial-NoDerivs 4.0 International License (CC BY-NC-ND 4.0), which permits the noncommercial replication and distribution of the article with the strict proviso that no changes or edits are made and the original work is properly cited (including links to both the formal publication through the relevant DOI and the license). See: https://creativecommons.org/licenses/by-nc-nd/4.0/.

\section{References}

1. China's seventh census. Available online: http://www.stats. gov.cn/tisj/zxfb/202105/t20210510_1817176.html

2. Lee D, Kim S, Kim K. International R\&D Collaboration for a Global Aging Society: Focusing on Aging-Related National-Funded Projects. Int J Environ Res Public Health 2020;17:8545.

3. Huang Y, Wang Y, Wang H, et al. Prevalence of mental disorders in China: a cross-sectional epidemiological study. Lancet Psychiatry 2019;6:211-24.

4. van Oort S, Beulens JWJ, van Ballegooijen AJ, et al. Association of Cardiovascular Risk Factors and Lifestyle Behaviors With Hypertension: A Mendelian
Randomization Study. Hypertension 2020;76:1971-9.

5. Stewart RAH, Held C, Krug-Gourley S, et al. Cardiovascular and Lifestyle Risk Factors and Cognitive Function in Patients With Stable Coronary Heart Disease. J Am Heart Assoc 2019;8:e10641.

6. Olesen KK, Madsen M, Würtz M, et al. Risk of Myocardial Infarction in Patients Without Angiographic Coronary Artery Disease Compared With the General Population. Am J Cardiol 2020;132:8-14.

7. Ziaeian B, Fonarow GC. Epidemiology and aetiology of heart failure. Nat Rev Cardiol 2016;13:368-78.

8. Cruz-Jentoft AJ, Bahat G, Bauer J, et al. Sarcopenia: revised European consensus on definition and diagnosis. Age Ageing 2019;48:16-31.

9. Bloom I, Shand C, Cooper C, et al. Diet Quality and Sarcopenia in Older Adults: A Systematic Review. Nutrients 2018;10:308.

10. Scherbakov N, von Haehling S, Anker SD, et al. Stroke induced Sarcopenia: muscle wasting and disability after stroke. Int J Cardiol 2013;170:89-94.

11. Bozzetti F. Chemotherapy-Induced Sarcopenia. Curr Treat Options Oncol 2020;21:7.

12. Philippou A, Xanthis D, Chryssanthopoulos C, et al. Heart Failure-Induced Skeletal Muscle Wasting. Curr Heart Fail Rep 2020;17:299-308.

13. Tournadre A, Vial G, Capel F, et al. Sarcopenia. Joint Bone Spine 2019;86:309-14.

14. Vellas B, Fielding RA, Bens C, et al. Implications of ICD-10 for Sarcopenia Clinical Practice and Clinical Trials: Report by the International Conference on Frailty and Sarcopenia Research Task Force. J Frailty Aging 2018;7:2-9.

15. Bielecka-Dabrowa A, Ebner N, Dos Santos MR, et al. Cachexia, muscle wasting, and frailty in cardiovascular disease. Eur J Heart Fail 2020;22:2314-26.

16. Zhao D, Liu J, Wang M, et al. Epidemiology of cardiovascular disease in China: current features and implications. Nat Rev Cardiol 2019;16:203-12.

17. Liu P, Hao Q, Hai S, et al. Sarcopenia as a predictor of all-cause mortality among community-dwelling older people: A systematic review and meta-analysis. Maturitas 2017;103:16-22.

18. Springer J, Springer JI, Anker SD. Muscle wasting and sarcopenia in heart failure and beyond: update 2017. ESC Heart Fail 2017;4:492-8.

19. Wu PY, Huang KS, Chen KM, et al. Exercise, Nutrition, and Combined Exercise and Nutrition in Older Adults with Sarcopenia: A Systematic Review and Network Meta- 
analysis. Maturitas 2021;145:38-48.

20. Lewison G. Preparation of bibliometrics papers. An Acad Bras Cienc 2020;92:e20201327.

21. Chen LK, Liu LK, Woo J, et al. Sarcopenia in Asia: consensus report of the Asian Working Group for Sarcopenia. J Am Med Dir Assoc 2014;15:95-101.

22. Dent E, Morley JE, Cruz-Jentoft AJ, et al. International Clinical Practice Guidelines for Sarcopenia (ICFSR): Screening, Diagnosis and Management. J Nutr Health Aging 2018;22:1148-61.

23. Cruz-Jentoft AJ, Sayer AA. Sarcopenia. Lancet 2019;393:2636-46.

24. Mensah GA, Roth GA, Fuster V. The Global Burden of Cardiovascular Diseases and Risk Factors: 2020 and Beyond. J Am Coll Cardiol 2019;74:2529-32.

25. Dennison EM, Sayer AA, Cooper C. Epidemiology of sarcopenia and insight into possible therapeutic targets. Nat Rev Rheumatol 2017;13:340-7.

26. Schneider SM, Correia MITD. Epidemiology of weight loss, malnutrition and sarcopenia: A transatlantic view. Nutrition 2020;69:110581.

27. Öztürk ZA, Türkbeyler İH, Abiyev A, et al. Health-related quality of life and fall risk associated with age-related body composition changes; sarcopenia, obesity and sarcopenic obesity. Intern Med J 2018;48:973-81.

28. Lim SK, Beom J, Lee SY, et al. Association between sarcopenia and fall characteristics in older adults with fragility hip fracture. Injury 2020;51:2640-7.

29. Anker SD, Morley JE, von Haehling S. Welcome to the ICD-10 code for sarcopenia. J Cachexia Sarcopenia Muscle 2016;7:512-4.

Cite this article as: Tan Z, Zhao Y, Jin Z, Li G, Xu L, Li W, Liang Y, Wang S, Zhu Q. The relationship between muscular atrophy/sarcopenia and cardiovascular diseases in the elderly: a bibliometrics study. Ann Palliat Med 2021;10(8):9136-9148. doi: 10.21037/apm-21-2144
30. Ryan E, McNicholas D, Creavin B, et al. Sarcopenia and Inflammatory Bowel Disease: A Systematic Review. Inflamm Bowel Dis 2019;25:67-73.

31. Peterson SJ, Mozer M. Differentiating Sarcopenia and Cachexia Among Patients With Cancer. Nutr Clin Pract 2017;32:30-9.

32. Choi CJ, Choi WS, Kim CM, et al. Risk of Sarcopenia and Osteoporosis in Male Tuberculosis Survivors: Korea National Health and Nutrition Examination Survey. Sci Rep 2017;7:13127.

33. Norman K, Otten L. Financial impact of sarcopenia or low muscle mass - A short review. Clin Nutr 2019;38:1489-95.

34. Graham H, White PC. Social determinants and lifestyles: integrating environmental and public health perspectives. Public Health 2016;141:270-8.

35. Meyer F, Valentini L. Disease-Related Malnutrition and Sarcopenia as Determinants of Clinical Outcome. Visc Med 2019;35:282-91.

36. Batsis JA, Villareal DT. Sarcopenic obesity in older adults: aetiology, epidemiology and treatment strategies. Nat Rev Endocrinol 2018;14:513-37.

37. Curcio F, Testa G, Liguori I, et al. Sarcopenia and Heart Failure. Nutrients 2020;12:211.

38. Lena A, Anker MS, Springer J. Muscle Wasting and Sarcopenia in Heart Failure-The Current State of Science. Int J Mol Sci 2020;21:6549.

39. Yin J, Lu X, Qian Z, et al. New insights into the pathogenesis and treatment of sarcopenia in chronic heart failure. Theranostics 2019;9:4019-29.

(English Language Editor: J. Teoh) 